\title{
Efficiency and Microbiological Substantiation of the Use of Er:YAG and Nd:YAG Lasers in Patients with Inflammatory Diseases of the Peri-Implant and Periodontal Tissues
}

\author{
Renat Garipov 1(D), Albert Davtyan ${ }^{1} \mathbb{D}$, Ekaterina Diachkova ${ }^{1, * \mathbb{D}}$, Maria Volkova ${ }^{1 \mathbb{C}}$, Svetlana \\ Repina ${ }^{1}$ (D), Svetlana Tarasenko ${ }^{1(D)}$
}

1 Department of Oral Surgery, E.V. Borovsky, Institute of Dentistry of I.M. Sechenov First Moscow State Medical University (Sechenov University), Russian Federation, Moscow; renatius88@mail.ru (R.G.); vantady@ yandex.ru (A.D.); secu2003@mail.ru (E.D.); yablokoland@yahoo.com (M.V.); lustra@bk.ru (S.R.); prof_tarasenko@ rambler.ru (S.T.)

* Correspondence: secu2003@mail.ru;

Received: 17.09.2020; Revised: 7.10.2020; Accepted: 9.10.2020; Published: 13.10.2020

\begin{abstract}
Due to the high incidence of inflammatory diseases of periodontal and peri-implant tissues, the problems of effective treatment of dental patients, reduction of the invasiveness of surgical interventions, and improvement of the course of the postoperative period remain urgent. In order to improve the effectiveness of treatment of patients in these categories, we conducted a study in which we analyzed the results of treatment of 100 patients with generalized periodontitis and peri-implantitis using traditional surgical techniques and with neodymium and erbium lasers. We used a microbiological research method to study changes in the quantitative and qualitative composition of the microbiota before and after the operations. The results of quantitative monitoring of the microbiota of the postoperative wound carried out in our study indicate that the dynamics of contamination with the use of traditional surgical and laser technologies are fundamentally different. With the traditional technique of surgical intervention, the maximum level of microbial colonization on day 3 was $8.2 \pm 0.4 \lg \mathrm{CFU}$, which creates a risk of developing purulent-inflammatory complications, while when using laser technologies, the microbial colonization during these periods was significantly less than after traditional surgical operations: $4.4 \pm 0.3 \mathrm{lg}$ CFU - when using the Nd: YAG laser and $5.9 \pm 0.4$ - when using the Er: YAG laser. This level of colonization is within the normal range of resident (normal) microbiota on the oral mucosa.
\end{abstract}

Keywords: periodontitis; peri-implantitis; neodymium laser; erbium laser; bacteriological research; microbiota; periodontal pocket.

(C) 2020 by the authors. This article is an open-access article distributed under the terms and conditions of the Creative Commons Attribution (CC BY) license (https://creativecommons.org/licenses/by/4.0/).

\section{Introduction}

Inflammatory periodontal diseases are among the most common, both in Russia and abroad $[1,2]$. According to the WHO, severe periodontitis occurs in 15-20\% of patients aged 35 to 44 years all over the world.

The widespread introduction of dental implant-supported prosthetics into dental practice has led to an increase in the number of patients with peri-implantation mucositis and peri-implantitis, which represent the inflammatory diseases of the peri-implant tissues. They 
are similar to gingivitis and periodontitis but differ in a number of features that should be taken into account when diagnosing, treating, and predicting the outcome of the disease [3].

Surgical techniques for the treatment of patients with inflammatory diseases of the periodontal and peri-implant tissues are widely described in the literature. However, the difficulties of performing surgical manipulations are in the conditions of insufficient visibility of the operating field due to bleeding as a result of the use of special cutting and rotary instruments $[4,5]$. On the other hand, antibiotic therapy, which is part of the treatment complex in outpatient surgery, is carried out using antimicrobial chemotherapeutic drugs that affect not only the pathogenic but also the resident microflora of the oral cavity and cause the development of resistance of periodontal pathogens to antibiotics [4, 6, 7]. As a result, in the postoperative period, clinically, pronounced edema, pain syndrome, a long healing period of soft tissues, and the possibility of the formation of rough scars are noted [8].

In recent years, new technologies have been actively introduced into medical practice, including treatment with laser radiation. [9-11]. The advantages of laser radiation are short duration and selectivity of exposure, which makes it less traumatic. This, in its turn, contributes to a shortening of the phase of inflammation, namely, earlier proliferation, which clinically determines a decrease in the severity of pain syndrome, collateral edema, and the formation of soft elastic scars. According to a number of authors, laser radiation has a pronounced antimicrobial effect [12-14]. The literature describes the positive results of the use of the erbium laser in periodontology $[8,13]$ and in the treatment of patients with peri-implantitis $[15$, 16]. A few works are dedicated to the combined use of erbium and neodymium lasers in periodontology $[17,18]$.

There are significantly fewer publications dedicated to the use of a neodymium laser. Thus, the results of a retrospective study by Italian authors, who studied the effectiveness of the use of neodymium laser radiation in the treatment of patients with periodontitis according to the PerioBLAST protocol (biological periodontal therapy using a laser) were presented [10]. Another promising neodymium laser treatment protocol for periodontal disease, proposed by Gregg and McCarthy in 1990, is LANAP, a procedure for creating a new epithelial attachment using a laser $[19,20]$. It should be noted that the neodymium laser has the deepest soft tissue penetration of all dental lasers. Scientists managed to develop a dental neodymium laser not so long ago. At the same time, such advantages of the laser as the ability to act not only on water but also on proteins, especially hemoglobin, are preserved. However, an in-depth analysis of the impact of studying the neodymium laser, including the KTP laser, on the microbiota of periodontal and peri-implant pockets has not been carried out.

Thus, it is of interest to study the antibacterial efficiency of neodymium laser radiation in the surgical treatment of patients with inflammatory diseases of the periodontal and periimplant tissues.

\section{Materials and Methods}

On the basis of the Department of Oral Surgery of the First Moscow State Medical University named after I.M. Sechenov and a private profile clinic, in the period from 2013 to 2018, we examined and treated 104 patients with inflammatory diseases of periodontal and peri-implant tissues: 72 patients with chronic generalized periodontitis of moderate and high severity and 32 patients with peri-implantitis, which developed on different terms after the placement of dental implants. All patients were randomly divided into 3 groups according to the method of treatment: 
I - treatment with a neodymium laser (Nd: $\mathrm{YAG})$;

II - treatment with an erbium laser (Er: YAG);

III - traditional surgical treatment.

The age of the patients in the groups corresponded to the WHO aged categories of 3140 and 41-50 years and was not statistically different across the comparison groups.

Patients of groups I and II underwent treatment of periodontal pockets and the area around the implants with neodymium and erbium lasers. Patients of group III (control group) traditional periodontal and outpatient surgical treatment.

All patients underwent clinical, radiological, and microbiological examination, including pre- and postoperative examination with the determination of the periodontal index; qualitative and quantitative analysis of the composition of the microflora of the periodontal pocket and peri-implant pocket using the bacteriological method and PCR diagnostics; radiation research methods for diagnostics and control of bone tissue regeneration; assessment of the effect of radiation of various types of laser on the surface of titanium implants using scanning electron microscopy.

To assess the effectiveness of the use of different technologies, we carried out a quantitative and qualitative assessment of the composition of the microbiota of the surgical wound before and after surgery on the 3rd, 5th, and 7th days. For fast and high-quality monitoring in the dynamics of the postoperative period, we, for the first time in operations of this type, used the apparatus for circular seeding and automated recording of results Scan500 (Interscience, USA).

For microscopic examination, we used traditional technologies of light microscopy under immersion. From the test material and isolated pure cultures, we gathered preparations that were fixed in the flame of an alcohol lamp and stained according to H. Gram's method, using aniline dyes (gentian violet, fuchsin), Lugol's solution, and 96\% ethanol to discolor smears.

Statistical processing was carried out by the method of nonparametric statistics, according to Mann - Whitney, with the determination of the probability of differences in the pvalue, taking into account the obtained middle values, middle errors, and sample sizes. The significance of differences in the frequency of detecting microorganisms was calculated using the nonparametric $\chi 2$ test with Yates correction for sample continuity. Values of $p<0.05$ were taken as a significant difference.

\section{Results and Discussion}

The average data of the quantitative dissemination of exudate taken with a standard swab from the surface of the surgical wound of the mucous membrane, expressed in terms of the decimal logarithm, ranged from 6.5 to 3.0 in the normal course of the postoperative period (106-103 CFU).

The research results obtained in the comparison groups are presented in table 1.

The initial contamination of exudate in patients did not differ by groups and correspond to the normal level of 104-7 CFU. In group I, immediately after the intervention, the microbial contamination of $5.7 \mathrm{lg}$ CFU was significantly lower than before. Later, and this was typical for the postoperative period after the application of the $\mathrm{Nd}$ : YAG laser, a statistically significant decrease in microbial contamination was observed, which stabilized on the 7 th day at the level of $3.5 \lg$ CFU. 
Table 1. The quantitative composition of the microbiota of the surgical wound (lg CFU).

\begin{tabular}{l|l|l|l|l|l} 
Group & $\begin{array}{l}\text { Before surgical } \\
\text { treatment }\end{array}$ & $\begin{array}{l}\text { After surgical } \\
\text { treatment }\end{array}$ & 3d day & 5th day & 7th day \\
\hline I & $6,5 \pm 0,4$ & $5,7 \pm 0,3^{*}$ & $4,4 \pm 0,3^{*}$ & $3,5 \pm 0,2^{*}$ & $3,5 \pm 0,3$ \\
\hline II & $6,35 \pm 0,5$ & $6,4 \pm 0,4$ & $5,9 \pm 0,4^{*}$ & $4,5 \pm 0,4^{*}$ & $4,3 \pm 0,3$ \\
\hline III & $6,25 \pm 0,5$ & $6,8 \pm 0,4$ & $8,2 \pm 0,4^{*}$ & $5,4 \pm 0,3^{*}$ & $4,9 \pm 0,3^{*}$ \\
\hline $\begin{array}{l}\text { Significance of intergroup } \\
\text { differences p }\end{array}$ & $>0,05$ & $\begin{array}{l}\text { pI-III }<0,05 \\
\text { pII-III }<0,05\end{array}$ & $\begin{array}{l}\text { pI-III }<0,02 \\
\text { pII-III }<0,02 \\
\text { pI-II }<0,05\end{array}$ & $\begin{array}{l}\text { pI-III }<0,02 \\
\text { pII-III }<0,02 \\
\text { pI-II }<0,05\end{array}$ & $\begin{array}{l}\text { pI-III-III }<0,025 \\
\text { pI-II }<0,005\end{array}$
\end{tabular}

*reliable difference from the value in the previous period.

In group II, a statistically significant decrease in microbial contamination to $5.9 \lg \mathrm{CFU}$ was achieved on day 3, but it was significantly higher than in group I. On the 5th day, there was a further decrease in microbial contamination to $4.5 \mathrm{lg}$ CFU, which was again significantly higher than in group I.

On the 7th day, the microbial count stabilized at almost the same level and amounted to $4.9 \lg$ CFU.

In group III, immediately after surgery, the exudate contamination of $6.8 \mathrm{lg}$ CFU was statistically significantly higher than in groups I and II, where laser technology was used. On the 3rd day, the microbial contamination increased sharply to $8.2 \mathrm{lg}$ CFU. Further, as the inflammatory reaction subsided, a statistically significant decrease in the degree of microbial contamination was observed, but nevertheless, they were statistically significantly higher than when using laser technologies.

Considering the important role of the qualitative or species composition of the microbiota, in particular, the fact that different species may differ in their pathogenic potential or drug resistance, during the monitoring, we dwelt on some features that could be traced from the results of the identification of isolated strains of microorganisms (Table 2).

During the operation, representatives of 17 major taxon were isolated from patients, among which were the main inhabitants of the oral cavity performing stabilizing functions in the microbiocenosis-streptococci (mainly $S$. sanguinis and $S$. mitis/oralis group) and diphtheroid (Corynebacterium sp.). They accounted for 30.7 and $7.7 \%$, respectively. A significant place in the composition of the microbiocenosis was occupied by gram-positive anaerobes, which accounted for $23.1 \%$. The most aggressive of these are peptostreptococci, peptococci, and clostridia. The representation of gram-negative anaerobes was also significant - 15.4\%, among which there were aggressive species of the periodontal pathogenic group: Fusobacterium sp., $P$. gingivalis and $P$. intermedia.

Quite high (13.9\%) was the proportion of enterococci and enterobacteria, which are capable of causing severe inflammatory complications of surgical interventions. In $9.2 \%$ of cases, that is, in every tenth patient, yeast fungi of the genus Candida were determined.

After performing surgical operations and appropriate treatment, the picture of the representation of the microbial flora changed significantly. Moreover, the number of isolated strains decreased from 65 to 25 in group I, to 17 in group II, and to 43 strains in the control group.

Correspondingly, the share of the main taxa in the structure of the microbiocenosis of the surgical wound changed, which allowed us to predict the development of dysbiotic changes in the postoperative period during traditional surgical treatment with antibiotic therapy or when using surgical lasers. 
Table 2. Results of identification of microorganisms from a surgical wound during surgery and after treatment (frequency of isolation from material in\%).

\begin{tabular}{|c|c|c|c|c|}
\hline Taxon & $\begin{array}{l}\text { Before surgical } \\
\text { treatment }\end{array}$ & Group I & Group II & Group III \\
\hline Streptococci & $20,0-30,7$ & $12,0-48,0 * \#$ & $7,0-41,2$ & $18,0-41,7$ \\
\hline S. sanguinis & $14,0-21,5$ & $9,0-36,0$ & $7,0-41,2$ & $12,0-27,9$ \\
\hline S. mitis/oralis & $3,0-4,6$ & $2,0-8,0$ & - & $3,0-6,9$ \\
\hline S. intermedius & $3,0-4,6$ & $1,0-4,0$ & - & $3,0-6,9$ \\
\hline Diphtheroids & $5,0-7,7$ & $5,0-20,0 * \#$ & —* & $4-9,3$ \\
\hline Corynebacterium & $4,0-6,2$ & $5,0-20,0$ & - & $4,0-9,3$ \\
\hline Actinomyces $s p$ & $1,0-1,5$ & - & - & - \\
\hline Gram $(+)$ anaerobes & $15,0-23,1$ & $2,0-8,0$ & —* & $4,0-9,3$ \\
\hline Propionibacterium & $2,0-3,1$ & $1,0-4,0$ & - & $1,0-2,3$ \\
\hline P. niger & $4,0-6,2$ & - & - & - \\
\hline P. anaerobius & $3,0-4,6$ & $1,0-4,0$ & - & $2,0-4,6$ \\
\hline S. anaerobius & $2,0-3,1$ & - & - & $1,0-2,3$ \\
\hline Clostridium sp & $4,0-6,2$ & - & - & - \\
\hline Gram (-) anaerobes & $10,0-15,4$ & $1,0-4,0 * \#$ & $3,0-17,5^{*}$ & $4,0-9,3$ \\
\hline Fusobacterium sp. & $3,0-4,6$ & - & - & $1-2,3$ \\
\hline Leptotrichia sp. & $1,0-1,5$ & - & - & - \\
\hline$P$. gingivalis & $3,0-4,6$ & $1,0-4,0$ & $2,0-11,8$ & $3,0-6,9$ \\
\hline P. intermedia & $3,0-4,6$ & - & $1,0-5,9$ & $2,0-4,6$ \\
\hline Other bacteria & $9,0-13,9$ & $3,0-12,0 * \#$ & $5,0-29,4^{*}$ & $7,0-16,3$ \\
\hline Enterococcus sp. & $5,0-7,7$ & $3,0-12,0$ & $5,0-29,4$ & $7,0-16,3$ \\
\hline Enterobacteriaceae & $4,0-6,2$ & - & - & - \\
\hline Candida sp. & $6,0-9,2$ & $2,0-8,0 * \#$ & $2,0-11,8^{*}$ & $6,0-13,9$ \\
\hline Total & $65-100$ & $25-100$ & $17-100$ & $43-100$ \\
\hline
\end{tabular}

The significance of the differences according to the $\chi 2$ test is <0.05: * - compared with group III; \# - compared with group II. Lines 1-7 - predominantly aggressive species, Lines 8-23 - mainly stabilizing species.

During traditional surgical interventions (group III), the predominance of microaerophilic streptococci $(41.7 \%)$ was noted, diphtheroids were isolated $(9.3 \%)$, but the share of potentially aggressive species - anaerobes (18.6\%), other bacteria $(16,3 \%)$, yeast fungi $(13.9 \%)$.

After using the Nd: YAG laser, the dominant flora was stabilizing: $48 \%$ were microaerophilic streptococci, and 20\% were corynebacteria (diphtheroids). The share of representatives of aggressive anaerobic species accounted for $12 \%$ and the same - for other bacterial species. The number of cases of detection of candida fungi decreased, but their relative proportion remained practically unchanged.

After using the Er: YAG laser, the dominant flora was also microaerophilic streptococci, which accounted for $41.2 \%$; however, corynebacteria and almost all Gram (+) anaerobic species were absent. 
Accordingly, the relative frequency of Gram (-) anaerobes, other bacteria, and candida fungi turned out to be significantly higher than in the case of the Nd: YAG laser.

Considering the results of a qualitative study of the microbial flora, it can be concluded that the Er: YAG laser had the most severe effect on the microbiota of the surgical wound; however, this did not lead to the normalization of the species composition towards a decrease in the release of aggressive species. More than $2 / 3$ of taxa (12 out of 17) were not detected after the application of this type of laser, which can be regarded as the development of a dysbiotic shift in the oral mucosa.

The data we obtained on the nature of the effect of the niodine laser in comparison with the erbium laser on the microbiota of the oral cavity, allow us to give a full characterization of the features of the microbiocenosis of the wound under conditions of laser exposure, corresponding to the known literature data [12-14, 18], but for the first time allow us to substantiate the high efficiency of the niodine laser in terms of its antimicrobial action.

\section{Conclusions}

Thus, we can conclude that the most favorable from the point of view of the risk of developing possible dysbiotic changes or wound suppuration in the postoperative period are the results of exposure to the Nd: YAG laser, which demonstrated the maximum reduction in the proportion of aggressive Gram (-) anaerobic bacteria and fungi while maintaining more 1/2 taxa of resident microorganisms. A clear reduction in the number and frequency of isolation of periodontal pathogenic species was noted. The use of the Er: YAG laser was characterized by the development of a dysbiotic shift due to the eradication of not only aggressive but also, to a large extent, normal microbiota. In the control group, the number of preserved taxa was maximum, but at the same time, periodontopathogenic species remained, and the relative proportion of fungi of the genus Candida even increased.

\section{Funding}

This research received no external funding.

\section{Acknowledgments}

This research has no acknowledgment.

\section{Conflicts of Interest}

The authors declare no conflict of interest.

\section{References}

1. Kondratyev, S.A.; Turkina, A.; Franco, A.; Budina, T.V.; Ovsienko, A.G. Prevalence of oral diseases and the assessment of the simplified oral hygiene, decayed-missing-filling and community periodontal indices among inmates of the Russian Federation. Bioscience Journal 2019, 35, 666-673, https://doi.org/10.14393/BJv35n2a20198-43408.

2. Könönen, E.; Gursoy, M.; Gursoy, U.K. Periodontitis: a multifaceted disease of tooth-supporting tissues. $-J$ Clin Med. 2019, 8, https://doi.org/10.3390/jcm8081135.

3. Passariello, C.; Di Nardo, D.; Testarelli, L. Inflammatory periimplant diseases and the periodontal connection question. Eur J Dent. 2019, 13, 119—23, https://doi.org/10.1055/s-0039-1688525.

4. Chrcanovic, B.R.; Kisch, J; Albrektsson, T; Wennerberg, A. A retrospective study on clinical and radiological outcomes of oral implants in patients followed up for a minimum of 20 years. Clin Implant Dent Relat Res. 2018, 20, 199-207, https://doi.org/10.1111/cid.12571. 
5. Ausenda, F.; Rasperini, G.; Acunzo, R.; Gorbunkova, A.; Pagni, G. New perspectives in the use of biomaterials for periodontal regeneration. Materials (Basel) 2019, 12, https://doi.org/10.3390/ma12132197.

6. Ippolitov, E.; Ilyasova, S.; Akhmedov, G.; Arutyunyan, A.; Tsarev, V. Clinical and immunological monitoring of gingival fluid cytokines in patients with perimplantitis during photodynamic therapy. Medical alphabet 2020, 1, 15-18, https://doi.org/10.33667/2078-5631-2020-12-15-18.

7. Saquib, S.A.; AlQahtani, N.A.; Ahmad, I.; Kader, M.A.; Al Shahrani, S.S.; Asiri, E.A. Evaluation and comparison of antibacterial efficacy of herbal extracts in combination with antibiotics on periodontal pathobionts: an in vitro micro- biological study. Antibiotics (Basel) 2019, 8, https://doi.org/10.3390/antibiotics8030089.

8. Talmac, A.C.; Calisir, M.; Eroglu, E.G.; Ertugrul, A.S. Effects of Er, Cr:YSGG and diode lasers on clinical parameters and gingival crevicular fluid IL-1 $\beta$ and IL-37 levels in generalized aggressive periodontitis. Mediators Inflamm. 2019.

9. Maskurova, Y.V.; Lalieva, Z.V.; Risovannaya, O.N.; Gaivoronskaya, T.V. Clinical substantiation of complex application of antioxidants and laser therapy in treatment of periodontal diseases. Klinicheskaya stomatologiia 2019, 28-30, https://doi.org/10.37988/1811-153X_2019_1_28.

10. Tarasenko, S.V.; Shekhter, A.B.; Minaev, V.; Morozova, E.A.; Gutorova, A.M.; Zhuravlev, A.N. 0.97 And $1.94 \mathrm{Mkm}$ Wavelength Laser Radiation Effect On The Oral Mucous Membrane Regeneration In The Experiment. Photonics, Russia 2019. 13, 108-116, https://doi.org/10.22184/FRos.2019.13.1.108.116.

11. Razmara, F.; Ghazanfari, R.; Shabankare, G. Laser-assisted uncovering of dental implants. Journal of Craniomaxillofacial Research 2020. http://dx.doi.org/10.18502/jcr.v6i4.3285.

12. De Falco, D.; Di Venere, D.; Maiorano, E. An Overview of Diode Laser-Assisted Oral Surgery. Cureus 2020, 12, https://doi.org/10.7759/cureus.9297.

13. Maniangat Luke, A.; Mathew, S.; Majed Altawash, M.; Mohammed Madan, B. Laser: A Review with Their Applications In Oral Medicine. Journal of Lasers in Medical Sciences 2019, 10, 234-329, https://doi.org/10.22037/jlms.v10i4.23044

14. De Falco, D.; Di Venere, D.; Favia, G. Laser Vestibuloplasty for Peri-implant Gingiva Implementation in the Atrophic Mandible of a Medically Compromised Patient. Cureus 2020, 12, https://doi.org/10.7759/cureus.7349.

15. Wang, J.; Wang, Hom-Lay. Laser-Assisted Therapy for Peri-implant Diseases. Lasers in Oral and Maxillofacial Surgery 2020, 123-137, https://doi.org/10.1007/978-3-030-29604-9_11

16. Świder, K.; Dominiak, M.; Grzech-Leśniak, K.; Matys, J. Effect of different laser wavelengths on periodontopathogens in peri-implantitis: A review of in vivo studies. Microorganisms 2019, 7, https://doi.org/10.3390/microorganisms7070189.

17. Shang, J.; Gong, K.; Xu, Da-Peng; Sun, Li-Wei; Qu, Wei-Dong. The Nd:YAG Laser or Combined with Er:YAG Laser Therapy for Oral Venous Lakes. Photobiomodulation, Photomedicine, and Laser Surgery 2020, 38, https://doi.org/10.1089/photob.2018.4484.

18. Grzech-Leśniak, K.; Sculean, A.; Gašpirc, B. Laser reduction of specific microorganisms in the periodontal pocket using Er:YAG and Nd:YAG lasers: a randomized controlled clinical study. J Lasers Med Sci. 2018, 33, 1461-70, https://doi.org/10.1007/s10103-018-2491-z.

19. Jha, A.; Gupta, V.; Adinarayan, R. LANAP, periodontics and beyond: a review. J Lasers Med Sci. 2018, 9 , 76-81, https://doi.org/10.15171/jlms.2018.16

20. McCawley, T.; McCawley, M.; Rams, T. Immediate Effects of Laser-Assisted New Attachment Procedure (LANAP) on Human Periodontitis Microbiota. Journal of the International Academy of Periodontology 2018, 20, 163-171. 